\title{
La medida de la competencia transversal Planificación y Gestión del Tiempo en el Grado en Ingeniería de Telecomunicaciones, Sonido e Imagen y Doble Grado con Comunicación Audiovisual
}

\section{Lourdes Canós-Darós ${ }^{\mathrm{a}}$, Cristina Santandreu-Mascarell ${ }^{\mathrm{b}}$ y Anna Vidal-Melóc}

a'Departamento de Organización de Empresas, Escuela Politécnica de Superior de Gandia, Universitat Politècnica de València, loucada@omp.upv.es, bepartamento de Organización de Empresas, Escuela Politécnica de Superior de Gandia, Universitat Politècnica de València, crisanma@omp.upv.es y 'Departamento de Matemática Aplicada, Escuela Politécnica de Superior de Gandia, Universitat Politècnica de Valencia, avidal@mat.upv.es

\begin{abstract}
The design of the Degree and Master's study plans has been a challenge for managers, lecturers and students, regarding the new consideration in the evaluation of different transversal skills. In this paper we focus on the transversal skill CT12-Planning and time management, defined for all the degrees in Universitat Politècnica de València. We present descriptive, quantitative (calculating a fuzzy adequacy coefficient to an ideal) and qualitative results, based on the students' perception of the items that define this concrete transversal skill according to the norms. Data has been collected from the first grade students in Telecommunications Engineering, Sound and Image Degree and Double Degree of this title with Media Communication, both taught at the UPV Gandia Campus, in the 2018-2019 academic year. Likewise, the questionnaire designed to meet our objective was answered in a second moment by all students in the 2019-2020 academic year in order to contrast the evolution of behaviors related to time management based on their perception.
\end{abstract}

Keywords: transversal skill, assessment, fuzzy, time management, measure, planning.

\section{Resumen}

El diseño de los planes de estudio de Grado y Máster ha supuesto un reto para gestores, profesores y estudiantes en cuanto a la nueva consideración en la evaluación de diversas competencias transversales. En este trabajo nos centramos en la competencia transversal CT12Planificación y gestión del tiempo, definida para todos los títulos de la Universitat Politècnica de València. Presentamos resultados descriptivos, cuantitativos (calculando un coeficiente fuzzy de adecuación a un ideal) y cualitativos, basados en la percepción de los estudiantes acerca de los items que definen la competencia transversal de acuerdo con la norma de la universidad. Los datos han sido recogidos de los estudiantes de primero de Grado en Ingeniería de Telecomunicaciones, Sonido e Imagen y Doble Grado de este título con Comunicación Audiovisual, ambos impartidos en el Campus de Gandia de la UPV, en el curso 2018-2019. Así mismo, el cuestionario diseñado para cumplir con nuestro objetivo fue contestado en un segundo momento por todos los estudiantes en el curso 2019-2020 para poder contrastar la evolución de los comportamientos relacionados con la gestión del tiempo en base a su percepción.

Palabras clave: competencia transversal, evaluación, fuzzy, gestión del tiempo, planificación. 


\section{Introducción}

Los títulos universitarios actuales tienen su origen en la Declaración de Bolonia de 19 de junio de 1999, en la que se definieron los ECTS (European Credit Transfer System), el Suplemento al Título y la organización de las enseñanza en nivel de Grado y Máster. Además del cambio organizativo en los títulos, se introdujo la idea de la adquisición de competencias como aspecto nuclear en el diseño de los planes de estudio, sin dejar de lado los contenidos tradicionalmente considerados (Marin-Garcia et al., 2009). En España, en el Real Decreto 1393/2007, de 29 de octubre, por el que se establece la ordenación de las enseñanzas universitarias oficiales se afirma que "los planes de estudios conducentes a la obtención de un título deberán, por tanto, tener en el centro de sus objetivos la adquisición de competencias por parte de los estudiantes, ampliando, sin excluir, el tradicional enfoque basado en contenidos y horas lectivas."

Es por esto que, además de las competencias genéricas y específicas de cada título, en la Universitat Politècnica de València se han definido 13 competencias transversales que están presentes en todos los planes de estudio de la Universidad, sea cual sea su especialidad (ver una breve definición de cada una de ellas en Tabla 1).

Tabla 1. Competencias transversales UPV

\begin{tabular}{|c|c|}
\hline $\begin{array}{l}\text { CT-01. Comprensión } \\
\text { integración }\end{array}$ & $\begin{array}{l}\text { Demostrar la comprensión en integración del conocimiento tanto de la propia } \\
\text { especialización como en otros contextos más amplios }\end{array}$ \\
\hline $\begin{array}{l}\text { CT-02. Aplicación } \\
\text { pensamiento práctico }\end{array}$ & $\begin{array}{l}\text { Aplicar los conocimientos a la práctica, atendiendo a la información disponible, y } \\
\text { estableciendo el proceso a seguir para alcanzar los objetivos con eficacia y } \\
\text { eficiencia }\end{array}$ \\
\hline $\begin{array}{l}\text { CT-03. Análisis y resolución de } \\
\text { problemas }\end{array}$ & $\begin{array}{l}\text { Analizar y resolver problemas de forma efectiva, identificando y definiendo los } \\
\text { elementos significativos que los constituyen }\end{array}$ \\
\hline $\begin{array}{l}\text { CT-04. Innovación, creatividad } \\
\text { y emprendimiento }\end{array}$ & $\begin{array}{l}\text { Innovar para responder satisfactoriamente, y de forma original, a las necesidades y } \\
\text { demandas personales, organizativas y sociales, con el propósito de aportar valor, } \\
\text { con una actitud emprendedora }\end{array}$ \\
\hline CT-05. Diseño y proyecto & $\begin{array}{l}\text { Diseñar, dirigir, desarrollar y evaluar una idea de manera eficaz hasta concretarla } \\
\text { en un servicio o producto }\end{array}$ \\
\hline $\begin{array}{l}\text { CT-06. Trabajo en equipo y } \\
\text { liderazgo }\end{array}$ & $\begin{array}{l}\text { Trabajar y liderar equipos de forma efectiva para la consecución de objetivos } \\
\text { comunes de un grupo de personas, contribuyendo al desarrollo personal y } \\
\text { profesional de los mismos }\end{array}$ \\
\hline $\begin{array}{l}\text { CT-07. Responsabilidad ética, } \\
\text { medioambiental y profesional }\end{array}$ & $\begin{array}{l}\text { Actuar con responsabilidad ética, medioambiental y profesional ante uno mismo y } \\
\text { los demás }\end{array}$ \\
\hline CT-08. Comunicación efectiva & $\begin{array}{l}\text { Comunicarse de manera efectiva, tanto de forma oral como escrita, utilizando } \\
\text { adecuadamente los recursos necesarios y adaptándose a las características de la } \\
\text { situación y de la audiencia }\end{array}$ \\
\hline CT-09. Pensamiento crítico & $\begin{array}{l}\text { Desarrollar un pensamiento crítico que se interese por los fundamentos en los que } \\
\text { se asientan las ideas, acciones y juicios, tanto propios como ajenos }\end{array}$ \\
\hline $\begin{array}{l}\text { CT-10. Conocimiento de } \\
\text { problemas contemporáneos }\end{array}$ & $\begin{array}{l}\text { Identificar e interpretar los problemas contemporáneos en su campo de } \\
\text { especialización así como en otros campos del conocimiento }\end{array}$ \\
\hline $\begin{array}{l}\text { CT-11. } \\
\text { permanente }\end{array}$ & $\begin{array}{l}\text { Utilizar el aprendizaje de manera estratégica, autónoma y flexible, a lo largo de } \\
\text { toda la vida, en función del objetivo perseguido }\end{array}$ \\
\hline $\begin{array}{l}\text { CT-12. Planificación y gestión } \\
\text { del tiempo }\end{array}$ & $\begin{array}{l}\text { Planificar adecuadamente el tiempo disponible y programar las actividades } \\
\text { necesarias para alcanzar los objetivos tanto académico-profesionales como } \\
\text { personales }\end{array}$ \\
\hline CT-13. Instrumental específica & $\begin{array}{l}\text { Utilizar adecuadamente las herramientas actualizadas necesarias para la práctica } \\
\text { de la profesión }\end{array}$ \\
\hline
\end{tabular}


Nuestro interés se centra en la competencia transversal número 12, Planificación y Gestión del Tiempo, que consiste en "Planificar adecuadamente el tiempo disponible y programar las actividades necesarias para alcanzar los objetivos tanto académico-profesionales como personales". En Tortajada et al. (2015) se presenta una experiencia de evaluación de esta competencia para alumnos de primer curso estudiantes de química, analizando las destrezas requeridas para una gestión del tiempo eficaz. También en Marcén y Martínez-Caraballo (2012) se muestran resultados sobre el aprovechamiento del tiempo que dedican al estudio los alumnos de primer curso de empresa y economía en la Universidad de Zaragoza.

Como es bien sabido, las nuevas tecnologías han modificado nuestros comportamientos ante la gestión de nuestro tiempo. Por ejemplo, la lectura se vuelve más rápida y superficial, contestamos mensajes a la vez que estamos haciendo otra actividad, y estamos pendiente de nuestros contactos permanentemente (Gómez-Hernández, 2012). En este contexto, Romero y Barberá (2013) reflejan cómo la falta de planificación y la mala gestión del tiempo son consideradas como la principal dificultad en la formación a distancia en la Universitat Oberta de Catalunya (UOC). Es fundamental para los estudiantes adquirir una buena gestión del tiempo y aprender a planificar sus actividades para poder ser un buen profesional, pues cualquier puesto que suponga alguna tarea de gestión requiere de esta competencia (Canós-Darós et al., 2003; Reverón, 2015).

En este trabajo se presentan los resultados procedentes de la medición de la competencia transversal Planificación y Gestión del Tiempo en el Grado en Ingeniería de Telecomunicaciones, Sonido e Imagen (GISTSI) y del Doble Grado en Ingeniería de Sistemas de Telecomunicación, Sonido e Imagen más Comunicación Audiovisual (GISTSI+CAU) que se imparten en el Campus de Gandia de la Universitat Politècnica de València. Para ello, primero realizamos una breve contextualización teórica sobre la definición de la competencia transversal que nos ocupa en este trabajo, así como la descripción contextual de la realización de la experiencia. Posteriormente, explicaremos cómo ha sido la toma de datos y el cálculo de los resultados, para pasar a exponer las principales conclusiones extraídas del proceso y mostrar las referencias bibliográficas utilizadas.

\section{Objetivos}

El objetivo principal de este trabajo es mostrar los resultados de la evolución en la adquisición de la competencia transversal Planificación y Gestión del Tiempo en estudiantado cursando los primeros cursos del GISTSI y del Doble Grado GISTSI+CAU, titulaciones impartidas en el Campus de Gandia de la Universitat Politècnica de València (UPV).

Los objetivos secundarios asociados al objetivo principal son los siguientes:

- Presentar una metodología para la medida de la competencia transversal acorde con la evaluación institucional.

- Analizar los indicadores de la rúbrica de evaluación propuesta por la Universidad y utilizarlos para el diseño de un cuestionario.

- Realizar una valoración cualitativa de la adquisición de la competencia transversal.

- Implementar técnicas fuzzy para la medida de la competencia transversal en los estudiantes de primer curso.

- Interpretar los resultados obtenidos teniendo en cuenta el contexto de la asignatura. 


\section{Desarrollo de la investigación}

\subsection{Contextualización}

La experiencia se realiza en el primer curso de los citados Grados, participando dos asignaturas, Matemáticas 2 y Economía de la Empresa en el sector de las Telecomunicaciones. Matemáticas 2 es una asignatura anual punto de control de una de las 13 competencias transversales que ha fijado la UPV, en concreto la competencia CT-12 Planificación y gestión del tiempo. Economía de la Empresa en el sector de las Telecomunicaciones se imparte en el segundo semestre de primer curso y tiene una carga de 6 ECTS, siendo punto de control de las competencias transversales CT04-Innovación, cretividad y emprendimiento, CT06-Trabajo en equipo y liderazgo y CT12-Planificación y gestión del tiempo.

Tomando como referencia los estudiantes de Matemáticas 2, 54 fueron los matriculados aunque realmente 49 han seguido la asignatura regularmente ( 5 fueron los no presentados en el acta final). En esta experiencia han participado un total de 27 estudiantes, 8 alumnas (de las 15 matriculadas) y 19 alumnos.

Durante el segundo semestre del curso 2018-2019, se les pasa una encuesta (Tabla 2) al alumnado, primeramente en la asignatura de Matemáticas 2 y posteriormente se recoge el cuestionario de los no asistentes en la asignatura Economía de la Empresa en el sector de las Telecomunicaciones, aprovechando la complementariedad de las dos asignaturas. Estos datos permitieron tener una visión del estado inicial del alumnado. Posteriormente, durante el primer semestre del curso 2019-2020 se pactó con una profesora de segundo curso el nuevo pase del cuestionario inicial para, de esta forma, obtener datos sobre la posible mejora, o no, de los estudiantes en cuanto a los items de la citada competencia.

\subsection{Recolección de datos}

Para la recolección de datos se diseñó un cuestionario para pasar a los estudiantes basado en la rúbrica de la Universitat Politècnica de València propuesta para la evaluación de la competencia transversal Planificación y Gestión del Tiempo, como se ve en la Tabla 2. Los ítems a valorar se determinaron a partir de la rúbrica institucional proporcionada por el Instituto de Ciencias de la Educación (ICE, 2015), que fue realizada por profesores considerados expertos en el objeto de la competencia transversal. En total, se seleccionaron once indicadores sobre la gestión del tiempo.

Tabla 2. Cuestionario para la recogida de datos

\begin{tabular}{|c|c|c|c|c|c|}
\hline Tu Nombre & Val. cuantitativa & \multicolumn{4}{|c|}{ Valoración cualitativa } \\
\hline & De 0 a 10 & A & $\mathrm{B}$ & $\mathrm{C}$ & $\mathrm{D}$ \\
\hline 1. Soy puntual & & & & & \\
\hline 2. Traigo el material necesario para la clase & & & & & \\
\hline 3. Priorizo las actividades y tareas según su importancia & & & & & \\
\hline 4. Establezco plazos para las diferentes tareas & & & & & \\
\hline 5. Si es el caso, entrego los trabajos en el plazo acordado & & & & & \\
\hline 6. Si es el caso, entrego los trabajos con el formato solicitado & & & & & \\
\hline $\begin{array}{l}\text { 7. Realizo las tareas o ejercicios a desarrollar, en el orden } \\
\text { establecido sin saltarme pasos }\end{array}$ & & & & & \\
\hline 8. Dedico a los ejercicios un tiempo razonable & & & & & \\
\hline $\begin{array}{l}\text { 9. Ante un ejercicio cuya resolución pueda hacerse de varias } \\
\text { formas, elijo siempre la forma más sencilla y rápida }\end{array}$ & & & & & \\
\hline $\begin{array}{l}\text { 10. En caso de dudas, busco alternativas como preguntar a un } \\
\text { compañero, al profesor, consultar Internet, etc. }\end{array}$ & & & & & \\
\hline 11. Consulto dudas a lo largo del curso y no al final (examen) & & & & & \\
\hline
\end{tabular}

(c)) EY-NC-ND 2020, Universitat Politècnica de València

Congreso In-Red (2020) 
La evaluación numérica se realizó utilizando una escala del 0 al 10, siendo 0 el mínimo y 10 el máximo. La escala cualitativa se cumplimentó con el código $\mathrm{A}=$ Excelente-Ejemplar / $\mathrm{B}=\mathrm{Bien}-\mathrm{Adecuado} / \mathrm{C}=\mathrm{En}$ desarrollo / $\mathrm{D}=$ No alcanzado, pues estas son las valoraciones que propone la Universidad para cada competencia transversal.

Además, se añadió la pregunta de respuesta libre "¿Qué objetivos te has planteado relacionados con la CT12-Planificación y Gestión del Tiempo?”, para conocer más en profundidad la idea de los estudiantes sobre esta competencia transversal.

\subsection{Análisis de los datos}

En este trabajo presentamos varios tipos de análisis. En primer lugar, un análisis descriptivo de datos que refleja la puntuación recogida en los dos momentos de tiempo y, por tanto, si ha habido una mejora en los ítems de cada alumno o un empeoramiento en el comportamiento percibido. En segundo lugar, presentamos los resultados del análisis cuantitativo realizado a través del coeficiente de adecuación entre las respuestas de los estudiantes y un ideal de comportamientos fijado por las profesoras. En tercer lugar, realizamos un análisis cualitativo a través de las respuestas libres realizadas por los estudiantes a la pregunta sobre los objetivos planteados en cuanto a la planificación y gestión del tiempo.

\subsection{Cálculo de la adecuación entre la valoración de los estudiantes y la de las profesoras}

Para la medida de la competencia transversal a través de los ítems definidos en el apartado anterior, primero se construyó un ideal con la valoración de las profesoras, expuesto en la Tabla 3.

Tabla 3. Valoración ideal de las profesoras

\begin{tabular}{|l|c|}
\hline & Val. cuantitativa \\
\hline Soy puntual & De 0 a 10 \\
\hline Traigo el material necesario para la clase & 9 \\
\hline Priorizo las actividades y tareas según su importancia & 9 \\
\hline Establezco plazos para las diferentes tareas & 8,5 \\
\hline Si es el caso, entrego los trabajos en el plazo acordado & 10 \\
\hline Si es el caso, entrego los trabajos con el formato solicitado & 9,5 \\
\hline $\begin{array}{l}\text { Realizo las tareas o ejercicios a desarrollar, en el orden } \\
\text { establecido sin saltarme pasos }\end{array}$ & 9 \\
\hline Dedico a los ejercicios un tiempo razonable & 8 \\
\hline $\begin{array}{l}\text { Ante un ejercicio cuya resolución pueda hacerse de varias } \\
\text { formas, elijo siempre la forma más sencilla y rápida }\end{array}$ & 7,5 \\
\hline $\begin{array}{l}\text { En caso de dudas, busco alternativas como preguntar a un } \\
\text { compañero, al profesor, consultar Internet, etc. }\end{array}$ & 9 \\
\hline Consulto dudas a lo largo del curso y no al final (examen) & 8,5 \\
\hline
\end{tabular}

Como se puede observar, lo que entienden las profesoras que es una valoración ideal para cada uno de los ítems que conforma la competencia transversal no es siempre el máximo, esto es, 10 puntos, pues hay que tener en cuenta que la competencia se trabaja en asignaturas de cursos posteriores pero enmarcadas en el mismo nivel de adquisición. 
Finalmente, se analizó el ajuste entre los datos procedentes de los estudiantes y el ideal de las profesoras utilizando un índice de adecuación que se representa en la siguiente expresión (Canós-Darós y Liern, 2008; Canós-Darós et al., 2018; 2019).

$$
\mu_{\widetilde{\mathrm{I}}^{\Phi}}\left(\widetilde{\mathrm{P}}_{\mathrm{j}}^{\Phi}\right)=\frac{1}{\mathrm{n}} \sum_{\mathrm{i}=1}^{\mathrm{n}} \mu_{\widetilde{\mathrm{I}}^{\Phi}}^{\mathrm{x}_{\mathrm{i}}}\left(\widetilde{\mathrm{P}}_{\mathrm{j}}^{\Phi}\right),
$$

$$
\text { donde } \mu_{\mathrm{I}^{\phi}}^{\mathrm{x}_{\mathrm{i}}}\left(\widehat{\mathrm{P}}_{\mathrm{j}}^{\phi}\right)=\frac{\operatorname{long}\left(\left[\mathrm{b}_{\mathrm{x}_{\mathrm{i}}}^{1}, \mathrm{~b}_{\mathrm{x}_{\mathrm{i}}}^{2}\right] \cap\left[\mathrm{a}_{\mathrm{x}_{\mathrm{i}}}^{1}, \mathrm{a}_{\mathrm{x}_{\mathrm{i}}}^{2}\right]\right)}{\operatorname{long}\left(\left[\mathrm{b}_{\mathrm{x}_{\mathrm{i}}}^{1}, \mathrm{~b}_{\mathrm{x}_{\mathrm{i}}}^{2}\right] \bigcup\left[\mathrm{a}_{\mathrm{x}_{\mathrm{i}}}^{1}, \mathrm{a}_{\mathrm{x}_{\mathrm{i}}}^{2}\right]\right)}
$$

Los resultados se ordenaron de mayor a menor de forma que el estudiante con mayor coeficiente es el más similar al perfil o nivel de adquisición de la competencia transversal propuesto por las profesoras.

\section{Resultados}

\subsection{Análisis descriptivo}

Comenzamos el análisis descriptivo presentando a continuación, en la Tabla 4, ítem a ítem, qué estudiantes han experimentado una mejora notable y cuáles han empeorado comparando las dos encuestas tomadas en los dos cursos consecutivos.

\begin{tabular}{|c|c|c|}
\hline Items & $\begin{array}{c}\text { Estudiantes que } \\
\text { mejoran } \\
\text { notablemente }\end{array}$ & $\begin{array}{c}\text { Estudiantes que } \\
\text { empeoran } \\
\text { notablemente }\end{array}$ \\
\hline 1. Soy puntual & $1-11-17-23$ & $20-22-24-26$ \\
\hline 2. Traigo el material necesario para la clase & $2-9-14$ & 22 \\
\hline 3. Priorizo las actividades y tareas según su importancia & $10-12-15-17-19-23$ & $1-2-3-6-14-22-27$ \\
\hline 4. Establezco plazos para las diferentes tareas & $\begin{array}{l}4-9-11-12-15-17-20- \\
23-24-27\end{array}$ & $7-10-14-16-18-25$ \\
\hline 5. Si es el caso, entrego los trabajos en el plazo acordado & $12-13-19-25$ & $3-22-26$ \\
\hline $\begin{array}{l}\text { 6. Si es el caso, entrego los trabajos con el formato } \\
\text { solicitado }\end{array}$ & $10-15-19-25-26$ & $2-11-22$ \\
\hline $\begin{array}{l}\text { 7. Realizo las tareas o ejercicios a desarrollar, en el orden } \\
\text { establecido sin saltarme pasos }\end{array}$ & $\begin{array}{l}8-10-11-15-17-19- \\
24-25\end{array}$ & $3-4-7-8-21-26$ \\
\hline 8. Dedico a los ejercicios un tiempo razonable & $9-11-15-19-23-24$ & $2-4-8-16-17-26-27$ \\
\hline $\begin{array}{l}\text { 9. Ante un ejercicio cuya resolución pueda hacerse de varias } \\
\text { formas, elijo siempre la forma más sencilla y rápida }\end{array}$ & $6-10-15-17-23-24$ & $1-2-5-16-18-20-26$ \\
\hline $\begin{array}{l}\text { 10. En caso de dudas, busco alternativas como preguntar a } \\
\text { un compañero, al profesor, consultar Internet, etc. }\end{array}$ & $5-7-22-25$ & $\begin{array}{l}8-10-11-14-17-19-21- \\
23-26\end{array}$ \\
\hline $\begin{array}{l}\text { 11. Consulto dudas a lo largo del curso y no al final } \\
\text { (examen) }\end{array}$ & $14-15-18-24-25$ & $\begin{array}{l}2-3-7-8-10-13-16-17- \\
21-23-27\end{array}$ \\
\hline
\end{tabular}

Tabla 4. Estudiantes con mejoras notables o empeoramiento notable

Pueden observarse en la Tabla 4 los siguientes casos destacables para los items que a continuación detallamos:

- Establezco plazos para las diferentes tareas, 10 mejoras respecto a 6 estudiantes que empeoran. Este ítem tiene el máximo número de mejoras. Aunque en el ítem correspondiente a la 
realización de las tareas o ejercicios a desarrollar, en el orden establecido sin saltarme pasos hay 8 mejoras, el número de estudiantes que empeora es de 6 .

- $\quad \mathrm{Si}$ es el caso, entrego los trabajos con el formato solicitado, 5 mejoras y 2 estudiantes que empeoran

- En caso de dudas, busco alternativas como preguntar a un compañero, al profesor, consultar Internet, etc., de 4 estudiantes que mejoran, 9 de ellos empeoran.

- Consulto dudas a lo largo del curso y no al final (examen), de los 5 estudiantes que mejoran, 11 han empeorado respecto al curso anterior.

- Los dos últimos ítems, relacionados con las dudas del estudiantes, son los que mayor número de estudiantes que empeoran tienen, 9 y 11 respectivamente.

En la Tabla 5 se puede ver la evolución de cada uno de los estudiantes respecto al número de items en los que experimenta una mejora notable, o al contrario, comparando las respuestas cualitativas de cada estudiante. Se supone que la mejora es notable si la escala cualitativa del curso 2019-2020 es mejor que la del 2018-2019 y al contrario en caso de empeoramiento.

Tabla 5. Estudiantes con mejoras notables o empeoramiento notable

\begin{tabular}{|c|c|c|c|}
\hline Estudiantes & $\begin{array}{l}N^{o} \text { de items con } \\
\text { mejora notable }\end{array}$ & $\begin{array}{l}N^{o} \text { de items con } \\
\text { empeoramiento } \\
\text { notablemente }\end{array}$ & $\begin{array}{l}N^{o} \text { de items sin } \\
\text { cambio notable }\end{array}$ \\
\hline Estudiante 1 & 1 & 2 & 8 \\
\hline Estudiante 2 & 1 & 5 & 5 \\
\hline Estudiante 3 & & 4 & 7 \\
\hline Estudiante 4 & 1 & 2 & 8 \\
\hline Estudiante 5 & 1 & 1 & 9 \\
\hline Estudiante 6 & 1 & 1 & 9 \\
\hline Estudiante 7 & 1 & 3 & 7 \\
\hline Estudiante 8 & & 4 & 7 \\
\hline Estudiante 9 & 4 & & 7 \\
\hline Estudiante 10 & 4 & 2 & 5 \\
\hline Estudiante 11 & 4 & 2 & 5 \\
\hline Estudiante 12 & 3 & & 8 \\
\hline Estudiante 13 & 1 & 1 & 9 \\
\hline Estudiante 14 & 2 & 3 & 6 \\
\hline Estudiante 15 & 7 & & 4 \\
\hline Estudiante 16 & & 4 & 7 \\
\hline Estudiante 17 & 5 & 3 & 3 \\
\hline Estudiante 18 & 1 & 2 & 8 \\
\hline Estudiante 19 & 5 & 1 & 5 \\
\hline Estudiante 20 & 1 & 2 & 8 \\
\hline Estudiante 21 & & 3 & 8 \\
\hline Estudiante 22 & 1 & 5 & 5 \\
\hline Estudiante 23 & 5 & 2 & 4 \\
\hline Estudiante 24 & 5 & 1 & 5 \\
\hline Estudiante 25 & 5 & 1 & 5 \\
\hline Estudiante 26 & 1 & 6 & 4 \\
\hline Estudiante 27 & 1 & 3 & 7 \\
\hline
\end{tabular}


La medida de la competencia transversal Planificación y Gestión del Tiempo en el Grado en Ingeniería de Telecomunicaciones, Sonido e Imagen y Doble Grado con Comunicación Audiovisual

En la Figura 1 puede observarse que el rango del número de items que mejoran es $[1,7]$ y en el que empeoran $[1,6]$. Además destacan varios grupos de estudiantes:

- Grupo de estudiantes cuyo número de items de mejora es bueno y no empeoran en ninguno: estudiante 15 , el 9 y el 12, con 7,4 y 3 items en los que mejoran respectivamente.

- Grupo de 7 estudiantes cuyo número de items de mejora es bueno pero empeoran en otros, ordenados de mayor mejora a menor número de peores: estudiantes 19, 24 y 25 (solo empeoran en un solo ítem), estudiante 23, estudiante 17 y estudiantes 10 y 11.

- Respecto a los que más items tienen que empeoran, destacar a 6 estudiantes, los números 2, 3, 8, 16,22 y 26 que empeoran en 4, 5 o 6 items.

Otro dato importante es que la tercera parte del alumnado encuestado conserva el nivel en el máximo número de items, 8 y 9.

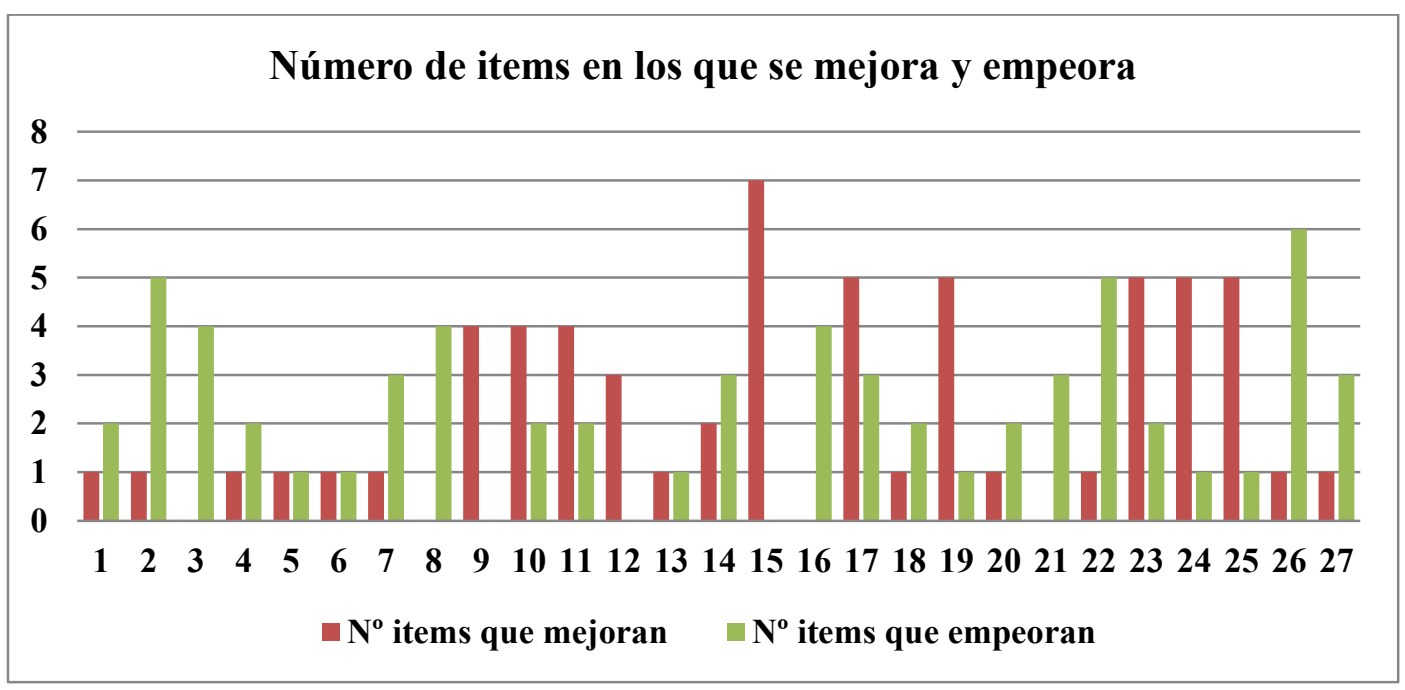

Fig. 1 Número de items por estudiante, mejores y peores

En la Figura 2 se observa el porcentaje de alumnado según el número de mejoras notables que se observan. Destaca que el 85,2\% mejoran en algún ítem, siendo lo más numeroso aquellos que mejoran un solo ítem, en el $44,4 \%$ o los que mejoran en 5 items con un $18,5 \%$. Los que no experimentan mejora en ninguno representan el $14,8 \%$.

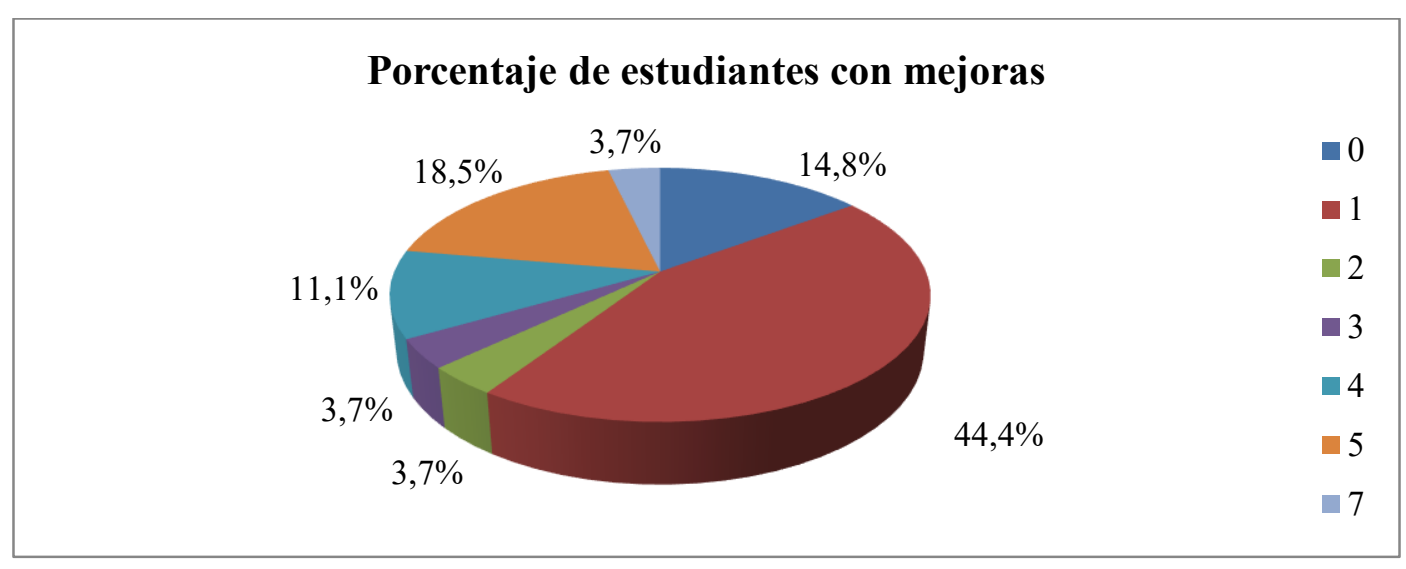

Fig. 2 Porcentaje alumnado según número de items de mejora notable

(c)) EY-NC-ND 2020, Universitat Politècnica de València

Congreso In-Red (2020) 
Respecto al alumnado que empeora destacan los que empeoran en 1, 2 o 3 items, sumando un total del $66,6 \%$, mientras que un $11 \%$ no empeora en ninguno, como se refleja en la Figura 3.

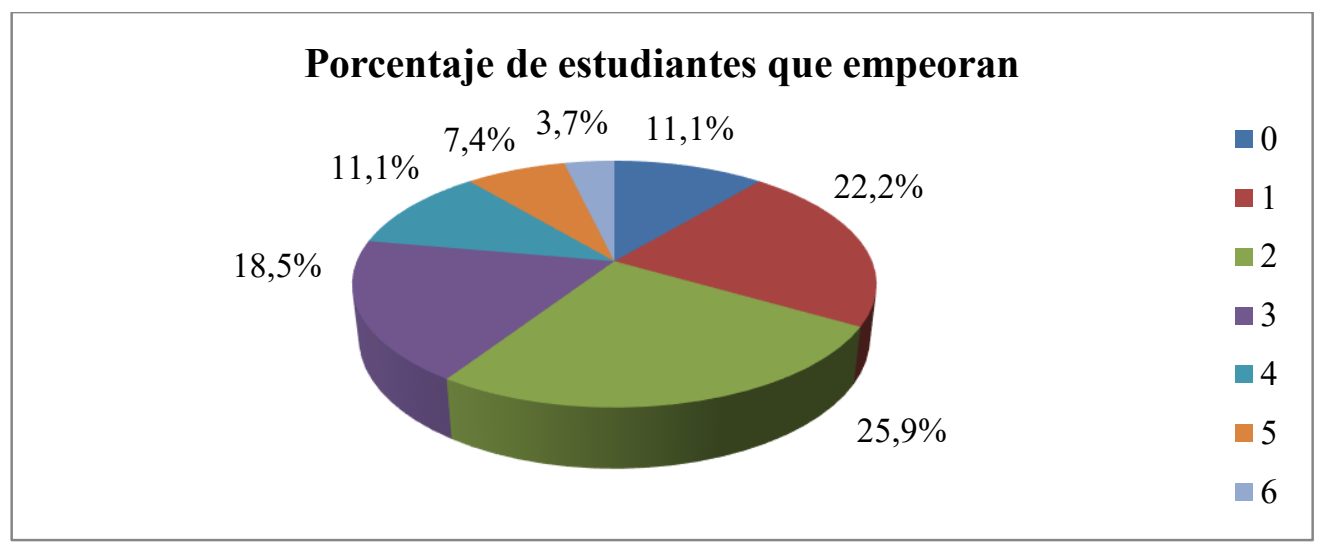

Fig. 3 Porcentaje alumnado según número de items que empeoran notablemente

La Figura 4 nos muestra si existe algún ítem en el que se mejora notablemente o viceversa teniendo en cuenta la totalidad de los estudiantes.

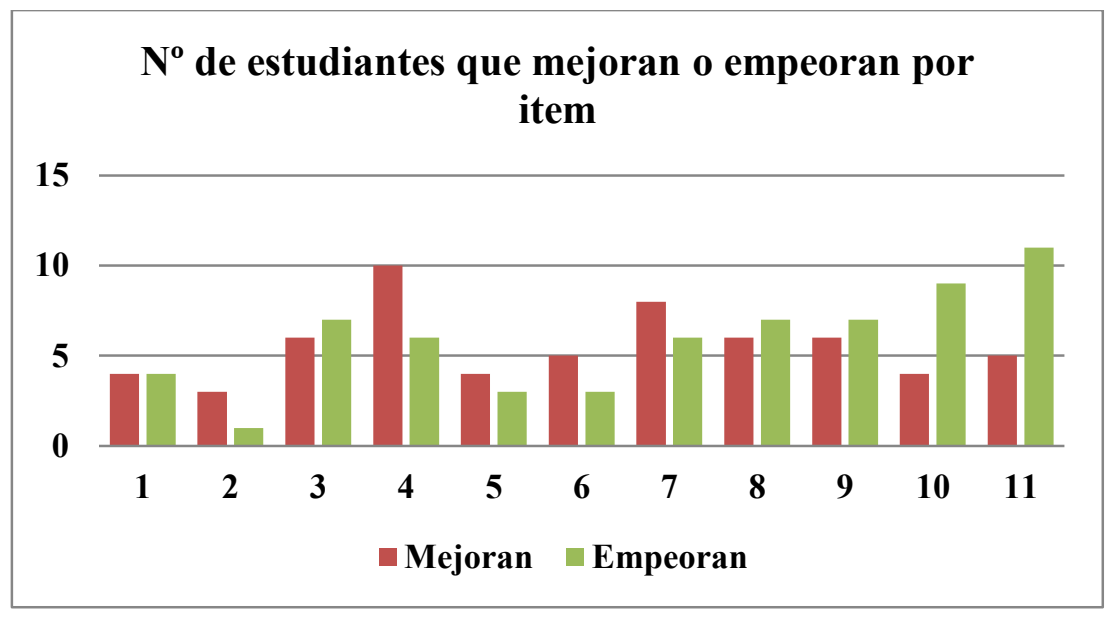

Fig. 4 Número de estudiantes que mejoran o empeoran por item

A la vista de la Figura 4 se observa que el ítem 4 "Establezco plazos para las diferentes tareas" es en el que más se ha mejorado (10 estudiantes), a pesar de los 6 que empeoran. Estableciendo la prioridad de mayor número de mejora, menos casos peores y diferencia entre ambos números positiva, se puede deducir, por orden, que lo ítems que han reflejado una mejora son:

- 4. Establezco plazos para las diferentes tareas

- 7. Realizo las tareas o ejercicios a desarrollar, en el orden establecido sin saltarme pasos

- 6. Si es el caso, entrego los trabajos con el formato solicitado

- 2. Traigo el material necesario para la clase

Los ítems que más han empeorado según la percepción de los estudiantes son el 10. En caso de dudas, busco alternativas como preguntar a un compañero, al profesor, consultar Internet, etc., y el 11. Consulto dudas a lo largo del curso y no al final (examen), ambos relacionados con la comunicación entre alumno y profesor y entre iguales.

(c)) BY-NC-ND 2020, Universitat Politècnica de València

Congreso In-Red (2020) 


\subsection{Análisis cuantitativo: el coeficiente de adecuación}

La formalización del coeficiente de adecuación utilizado en este trabajo se ha descrito en un apartado anterior. En definitiva, se trata de ver qué estudiantes están más cerca del ideal de alcance de la competencia transversal CT12-Planificación y gestión del tiempo descrito por las profesoras de primer curso.

Tabla 6. Resultados para los cursos 2018-2019 y 2019-2020

\begin{tabular}{|c|c|c|c|}
\hline \multicolumn{2}{|c|}{ Curso 2018-2019 } & \multicolumn{2}{|c|}{ Curso 2019-2020 } \\
\hline 10,00 & Estudiante 8 & 9,73 & Estudiante 4 \\
\hline 9,86 & Estudiante 4 & 9,64 & Estudiante 12 \\
\hline 9,77 & Estudiante 21 & 9,64 & Estudiante 25 \\
\hline 9,61 & Estudiante 3 & 9,36 & Estudiante 5 \\
\hline 9,50 & Estudiante 5 & 9,36 & Estudiante 9 \\
\hline 9,41 & Estudiante 1 & 9,36 & Estudiante 17 \\
\hline 9,41 & Estudiante 16 & 9,32 & Estudiante 8 \\
\hline 9,23 & Estudiante 18 & 9,23 & Estudiante 1 \\
\hline 9,18 & Estudiante 12 & 9,00 & Estudiante 3 \\
\hline 9,14 & Estudiante 15 & 8,95 & Estudiante 16 \\
\hline 9,09 & Estudiante 2 & 8,82 & Estudiante 21 \\
\hline 9,09 & Estudiante 17 & 8,82 & Estudiante 24 \\
\hline 8,95 & Estudiante 14 & 8,73 & Estudiante 2 \\
\hline 8,77 & Estudiante 13 & 8,68 & Estudiante 13 \\
\hline 8,77 & Estudiante 25 & 8,68 & Estudiante 14 \\
\hline 8,77 & Estudiante 27 & 8,68 & Estudiante 18 \\
\hline 8,59 & Estudiante 26 & 8,59 & Estudiante 11 \\
\hline 8,55 & Estudiante 22 & 8,45 & Estudiante 23 \\
\hline 8,45 & Estudiante 7 & 8,00 & Estudiante 7 \\
\hline 8,18 & Estudiante 24 & 7,91 & Estudiante 27 \\
\hline 8,09 & Estudiante 10 & 7,77 & Estudiante 10 \\
\hline 7,95 & Estudiante 11 & 7,23 & Estudiante 22 \\
\hline 7,00 & Estudiante 6 & 7,09 & Estudiante 20 \\
\hline 7,00 & Estudiante 23 & 7,00 & Estudiante 26 \\
\hline 6,86 & Estudiante 20 & 6,73 & Estudiante 6 \\
\hline 5,36 & Estudiante 19 & 6,73 & Estudiante 19 \\
\hline
\end{tabular}

Como podemos observar en la Tabla 6 existen dos estudiantes (estudiante 4 y estudiante 5) que se encuentran en los dos cursos considerados entre las cinco primeras posiciones, lo que significa que su carácter en cuanto a la competencia transversal de Planificación y Gestión del Tiempo es estable de acuerdo con el ideal de las profesoras, que no ha cambiado para los dos cursos académicos. En este orden de ideas, en el final de la tabla coinciden para los dos cursos los estudiantes 6,19 y 20, lo que indica que sufren una mala gestión del tiempo en general para la planificación de sus actividades.

Por otra parte, los estudiantes 8 y 21 que se encuentran en el curso 2019-2020 en primera y tercera posición respectivamente respecto al idea de adquisición de la competencia, en el siguiente curso descienden a los puestos 7 y 11; esto significa que han empeorado en la gestión global de la competencia transversal y sería conveniente averiguar por otros medios (entrevista, por ejemplo) qué ha sucedido para llegar a esta situación. En clave positiva, vemos que los estudiantes 11 y 23 que están en el curso 2019- 
2020 al final de la tabla han mejorado su ajuste con el ideal de adquisición de la competencia transversal marcado, pasando a ocupar puestos intermedios en la tabla correspondiente a los resultados del curso 2019-2020.

\subsection{Análisis cualitativo}

En el cuestionario diseñado para la obtención de datos se realizó una pregunta de respuesta abierta: “¿Qué objetivos te has planteado relacionados con la CT12-Planificación y Gestión del Tiempo?". Las respuestas más repetidas versaron sobre la planificación de las tareas y del estudio de la materia de todas las asignaturas del curso para no dejarlo todo al final y llegar a tiempo a las entregas, optimizar el tiempo de estudio y utilizar el calendario (trabajos, exámenes). Con estas respuestas, podemos pensar que verdaderamente la gestión del tiempo de las tareas, trabajos, estudio individual, etc., de todo el curso supone una preocupación para los estudiantes, que son conscientes de que no pueden dejar todo para un último momento.

\section{Conclusiones}

La adquisición de la competencia transversal Planificación y Gestión del Tiempo es fundamental para que el alumnado universitario tenga un buen desempeño en su futura vida laboral. Es por esto, que se ha de trabajar en el aula y de forma autónoma desde primer curso de Grado.

En este trabajo hemos presentado los resultados de la evaluación de la competencia transversal definida en la Universitat Politècnica de València tomando como referencia el estudiantado de primeros cursos del Grado en Ingeniería de Telecomunicaciones, Sonido e Imagen y del Doble Grado en Ingeniería de Sistemas de Telecomunicación, Sonido e Imagen más Comunicación Audiovisual, impartidos en el Campus de Gandia de dicha universidad.

Para ello, hemos mostrado una metodología de evaluación propia basada en la rúbrica institucional, en la que se ha considerado la autoevaluación de los estudiantes. Con esta información, por una parte, hemos analizado los datos de forma cualitativa basándonos en la norma que establece la Universidad y, por otra, hemos realizado un análisis descriptivo de datos y hemos calculado un coeficiente de adecuación que ha permitido conocer el ajuste entre los estudiantes y el ideal de adquisición de la competencia dado por las profesoras.

Con estos resultados hemos conocido mejor la evolución de los estudiantes en la adquisición de la competencia transversal Planificación y gestión del tiempo, analizando con detalle los casos en los que ha habido un empeoramiento en su valoración para un determinado estudiante. Como se ha indicado anteriormente, los ítems 10 y 11 del cuestionario realizado por los estudiantes, relacionados con la resolución de dudas, son los menos adquiridos por los estudiantes. A pesar del sistema de tutorías del que disponen los alumnos y que el profesorado debe cumplir, en general el estudiantado solo lo utiliza mayoritariamente para revisión de exámenes, siendo el uso correcto la resolución de dudas durante el curso. Por este motivo, se podría intuir que los alumnos no suelen llevar la asignatura al día, pudiendo ser el principal motivo por el que no se realiza el uso correcto de las tutorias. Para intentar una mejora y potenciar que el estudiantado lleve la asignatura al día, se proponen metodologías como preguntas del minuto al empezar cada una de las sesiones de clase, aplicar herramientas de gamificación como Kahoot! o Socrative y de esta manera motivarles en cuanto a la resolución de posibles dudas. En estos casos los profesores han de averiguar cuál es la causa de esta menor autovaloración y tratar de reconducir la situación en beneficio del estudiante. Otras acciones que podrían llevarse a cabo en la asignatura para 
La medida de la competencia transversal Planificación y Gestión del Tiempo en el Grado en Ingeniería de Telecomunicaciones, Sonido e Imagen y Doble Grado con Comunicación Audiovisual

mejorar el nivel de la competencia en los estudiantes son el intentar personalizar más la docencia citándolos en tutorías individuales, por ejemplo, usar alguna aplicación informática de gestión del tiempo (agenda, planificación de tareas, etc.), despertar su curiosidad científica con problemas reales, etc.

\section{Agradecimientos}

Las autoras quieren agradecer la ayuda económica y el apoyo institucional recibidos de la Universitat Politècnica de València a través de los proyectos PIME 19-20/186 y 19-20/190.

\section{Referencias}

CANOS-DAROS, L. y LIERN CARRION, V. (2008): "Soft computing-based aggregation methods for human resource management" en European Journal of Operational Research, vol. 189, núm. 3, p. 669-681.

CANOS-DAROS, L.; GUIJARRO, E. SANTANDREU-MASCARELL, C. y BABILONI, E. (2018). "Evaluación cualitativa y cuantitativa de la competencia transversal Trabajo en equipo y Liderazgo: una experiencia basada en la evaluación por pares y autoevaluación.“ En XXXII Annual Conference AEDEM, Gandia.

CANOS-DAROS, L.; GUIJARRO, E. SANTANDREU-MASCARELL, C. y BABILONI, E. (2019). "Evaluación por pares y autoevaluación de la competencia transversal trabajo en equipo" en Journal of Management and Business Education, vol. 2, núm. 2, p. 69-86.

DECLARACIÓN DE BOLONIA, 19 de junio de 1999.

GOMEZ-HERNANDEZ, J.A. (2013). "La gestión del tiempo como dimensión de la competencia informacional". Anuario ThinkEPI, vol. 7, p. 28-36.

ICE, Instituto de Ciencias de la Educación (2015). "Rúbrica CT12-Planificación y Gestión del Tiempo", Documento de trabajo, Universitat Politècnica de València.

MARCÉN, M. y MARTÍNEZ-CARABALLO, N. (2012). "Gestión eficiente del tiempo de los universitarios: evidencias para estudiantes de primer curso de la Universidad de Zaragoza" en INNOVAR. Revista de Ciencias Administrativas y Sociales, vol. 22, núm. 43, enero-marzo, p.117-130.

MARIN-GARCIA, J.A., GARCÍA SABATER, J.P., PERELlO MARÍN, M.R. y CANÓS DARÓS, L. (2009). "Propuesta de competencias para el Ingeniero de Organización en el contexto de los nuevos planes de estudios" en Intangible Capital, vol. 5, p. 387-406.

REAL DECRETO 1393/2007, de 29 de octubre, por el que se establece la ordenación de las enseñanzas universitarias oficiales.

REVERÓN SUÁREZ, N. (2015). "La gestión del tiempo (Time Management)“. Trabajo Fin de Grado. Universidad de La Laguna.

ROMERO, M y BARBERÀ, E. (2013). "Identificación de las dificultades de regulación del tiempo de los estudiantes universitarios en formación a distancia“" en Revista de Educación a Distancia, vol. 38.

TORTAJADA GENARO, L.A., HERRERO VILLÉN, M.A., NOGUERA MURRAY, P., MORAIS EZQUERRO, S. y ATIENZA BORONAT, J. (2015). “¿Cómo evaluaremos la competencia transversal "Planificación y gestión del tiempo" a un alumno de primer curso? " En Congreso In-Red 2015. Valencia: Universitat Politècnica de València, 114.

UPV, Universitat Politècnica de València (2012). Competencias Transversales UPV. Disponible en www.upv.es/contenidos/COMPTRAN 